\title{
Can We Diminish Spreading of the COVID-19 Pandemic?
}

\author{
S. Haber ${ }^{1}$, A. Tsuda ${ }^{2,3}$ \\ ${ }^{1}$ Department of Mechanical Engineering, Technion-Israel Institute of Technology, Haifa, Israel; ${ }^{2}$ Retired from HSPH, \\ Harvard University, Boston, MA, USA; ${ }^{3}$ Currently Tsuda Lung Research, Shrewsbury, MA, USA
}

Correspondence to: S. Haber, shimonhaber@gmail.com, mersh01@technion.ac.il;

A. Tsuda, atsuda@hsph.harvard.edu

Keywords: Bioaerosols, Surfactants, Lung Airways

Received: December 14, $2020 \quad$ Accepted: January 23, $2021 \quad$ Published: January 26, 2021

Copyright $\odot 2021$ by author(s) and Scientific Research Publishing Inc.

This work is licensed under the Creative Commons Attribution International License (CC BY 4.0).

http://creativecommons.org/licenses/by/4.0/

\section{(c) (i) Open Access}

\section{ABSTRACT}

We hypothesized that liquid menisci occlusions may form inside small airways, travel along the airway while losing mass and finally may disintegrate into bioaerosols. Spreading of the COVID-19 virus is strongly related to the number of such bioaerosols exhaled by "superspreaders". We show, employing numerical methods, that this number can be diminished by administering surfactants which lower the surface-tension of the mucus which covers the airways.

\section{INTRODUCTION}

It is well acknowledged that the main transmission mechanism of infection of SARS-COV-2 virions is inhalation [1] of droplets exhaled by a nearby sick person and asymptomatic COVID-19 positive individuals.

Applying numerical methods, we investigated [2] the mechanism of how infectious droplets can be formed at lesion during normal breathing. We hypothesized that liquid menisci occlusions may form inside small airways, travel along the airway while losing mass and finally may disintegrate into bioaerosols (Figure 1).

\section{ANALYSIS}

A major factor that controls a breakup is the capillary number $(\mathrm{Ca})$ of the mucus layer that covers the airway. The capillary number stands for the ratio between the hydrodynamic shear forces and the surface tension at the gas-liquid interface [2]. For capillary numbers lower than a critical value $\left(C a_{c r}\right)$ (i.e. high surface tension), no droplets are formed. Alas, the occlusion remains intact and grows with time. For capillary numbers higher than the critical value (i.e., low mucus surface tension) the menisci diminish in size and may form droplets (Figure 1).

The number and size of disintegrated droplets are a result of instability of the thinning meniscus 
(a) $\mathrm{Ca} / \mathrm{Ca} a_{c r}=1.13$

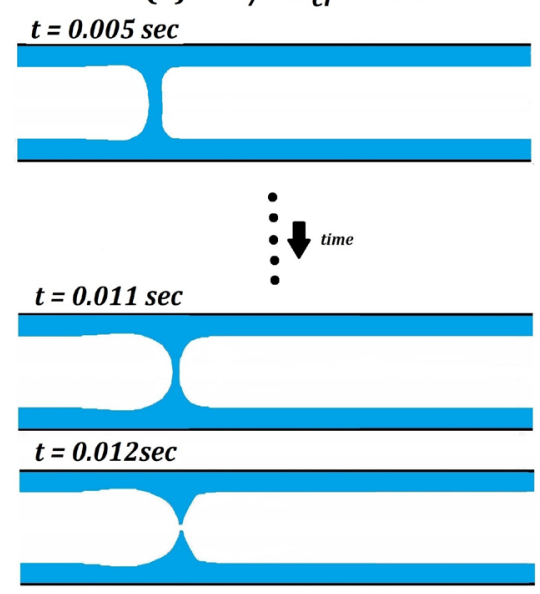

(b) $\mathrm{Ca} / \mathrm{Ca} a_{c r}=3.86$
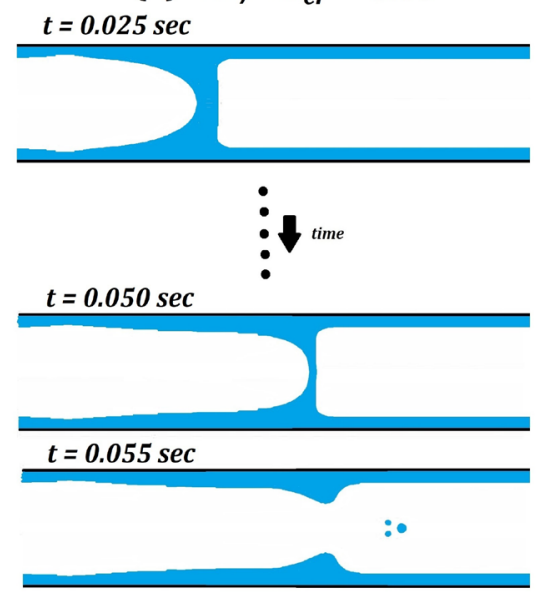

(c) $\mathrm{Ca} / \mathrm{Ca} \mathrm{cr}=7.64$

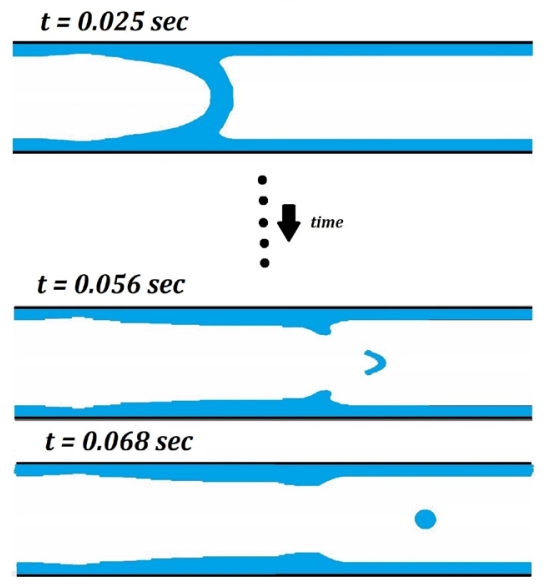

Figure 1. Meniscus disintegration modes: (a) at $C a / C a_{c r}=1.13$, meniscus collapses and no drops are formed; (b) at $C a / C a_{c r}=3.86$, three droplets are formed, (c) at $C a / C a_{c r}=7.64$, a single droplet is formed. (Adapted and modified from reformed Malashenko et al., 2009). See also a video animation in supplementary material.

layer. For $C a \approx C a_{c n}$ no droplets are created (Figure 1(a)). For $C a>C a_{c r}$ (i.e., small surface tension), a number of droplets can be generated, depending on the capillary number value (Figure 1(b) \& Figure 1(c)). In particular, for $C a \gg C a_{c r}$ (i.e., very small surface tension) only a single and relatively large droplet is generated (Figure $1(\mathrm{c})$ ).

It is well known [1] that also healthy people exhale bioaerosols during normal breathing/speaking. The number of exhaled droplets may vary and the individual, who emits a large number of droplets, is so called a "super-spreader" [3]. As a prevention, identifying the "super spreaders" and reducing the surface tension of their lung mucus would greatly minimize the number of droplets they exhale and, consequently, reduce the spreading of the coronavirus (and other viral lung related diseases) in case they become infected.

\section{SUMMARY}

We suggest a two-step procedure: 1) Identify super spreaders and 2) Administer a safe material which lowers lung mucus surface tension.

We believe that step 1) could be achieved applying well known methods. Much research regarding 2) is currently undertaken as finding a new nanomaterial as well as safe treatments of surfactant replacement therapy for premature babies and patients suffering from Acute Respiratory Distress Syndrome (ARDS). When we achieve the suggested two step procedure in the public, we could significantly minimize the spreading of pandemics [4].

\section{CONFLICTS OF INTEREST}

The authors declare no conflicts of interest regarding the publication of this paper.

\section{REFERENCES}

1. CDC Coronavirus Disease 2019 (COVID-19). https://www.cdc.gov/coronavirus/2019-ncov/more/scientific-brief-sars-cov-2.html

2. Malashenko, A., Tsuda, A. and Haber, S. (2009) Propagation and Breakup of Liquid Menisci and Aerosol Generation in Small Airways. Journal of Aerosol Medicine and Pulmonary Drug Delivery, 22, 341-353. 
https://doi.org/10.1089/jamp.2008.0696

3. CDC Coronavirus Disease 2019 (COVID-19). https://wwwnc.cdc.gov/eid/article/26/6/20-0495-t1

4. Centers for Disease Control and Prevention.

https://www.cdc.gov/media/dpk/diseases-and-conditions/coronavirus/coronavirus-2020.html 